\title{
Nonlinear Output Feedback Control for Robust Stability
}

\author{
Chengkang Xie and Mark French
}

\begin{abstract}
In the presence of input and measurement disturbances, an observer and a controller are designed to achieve robust stability for the nominal plant in normal form. By framework of nonlinear gap metric, a robust margin of plant perturbations is obtained, and the controller is shown to be robust to plant perturbations if the gap metric is less than the robust margin.
\end{abstract}

\section{INTRODUCTION}

In general, to control a physical plant, a mathematical model ( called the nominal plant ) for the plant is necessary. But, in practice, the nominal plant cannot completely describe the physical plant, there always exists a plant perturbation. In operation of control, sensors and actuators are setup to measure and input signals, and the sensors and actuators result in measurement errors and input errors, namely, measurement disturbances and input disturbances. A closed-loop could become unstable if a controller cannot tolerate these kinds of uncertainties. EI-Sakkary [1] gave an example that a small uncertainty changed the stability of the closed-loop. So, for control purposes, a basic requirement is that a controller designed for the nominal plant tolerates plant perturbations, measurement disturbances and input disturbances, that is the controller is robust to these kinds of uncertainties.

Although the study of robustness for control designs is as old as feedback control, even for linear systems effective systematic tools for robust control have only been developed since 1980's. An appropriate topological structure for studying the robustness of linear systems is the gap metric ( graph topology ) introduced by Zames and EI-Sakkary [8], [1]. In contrast, other frameworks for studying robustness have restrictions; e.g., the order of parametric uncertainty cannot be changed, a small time delay is not an allowable uncertainty, Un-modeled dynamics or plant perturbations are not allowed, etc.. For nonlinear control, it is difficult to cope with the complexity of nonlinear phenomena even in the absence of disturbances and other uncertainties, the robustness study of nonlinear systems is far less developed than for linear systems. In 1997, Georgiou and Smith [4] established a theory of gap metric for nonlinear case. It provides a powerful tool to study robustness of nonlinear

C. Xie is with Institute of Mathematics, Southwest University, Beibei, Chongqing, 400715, P.R. China; cxie@swu.edu.cn. The author was supported by the Natural Science Foundation of Chongqing under Grants CSTC 2005EB2048.

M. French is with School of Electronics and Computer Science, University of Southampton, Southampton, SO17 1BJ, UK mcfeecs.soton.ac.uk control. Via the framework of nonlinear gap metric, Xie and French [7] successfully designed a robust backstepping controller for state feedback in 2003. Any restrictions on disturbances and plant perturbations have been removed, and the disturbances and perturbations can be un-modeled dynamics. So, it presents that the framework of nonlinear gap metric is a appropriate topological structure for nonlinear feedback control.

In the past two decades, many of control techniques have been developed for nonlinear systems using feedback control. Most of the results, however, assume full state feedback. Efforts to extend some of these results to output feedback have naturally included the idea of designing an observer to estimate the state of a system from its output, see, e.g., [5], [2], [6], [3]. It is a meaningful work to utilize the framework of nonlinear gap metric for the study of output feedback control.

In this paper, we consider a nominal plant in normal form and design an observer and a controller to achieve robust stability of the closed-loop system in the presence of input and measurement disturbances. Then we utilize the results in [4] to obtain the robustness of the closed-loop to plant perturbations which are small in the sense of the gap metric. That is, we show that the controller stabilizes the closed-loop for any perturbed plant in the presence of input and measurement disturbances if the gap metric distance between the nominal and perturbed plant is less than a computable constant. Any restrictions on input and measurement disturbances, and plant perturbations have been removed, and the disturbances and perturbations can be unmodeled dynamics.

\section{BACKGROUND AND PROBLEM FORMULATION}

In this section we first simply state the background for gap metric robustness. The material here comes from the fundamental paper [4].

\section{A. Background for Gap Metric Robustness}

Let $\mathcal{U}$ and $\mathcal{Y}$ be normed signal spaces, and consider a causal nominal plant

$$
\mathcal{P}: \mathcal{U} \rightarrow \mathcal{Y}: u_{1} \mapsto y_{1}
$$

and a causal controller

$$
\mathcal{C}: \mathcal{Y} \rightarrow \mathcal{U}: y_{2} \mapsto u_{2}
$$

In a control system, there exist measurement disturbance $y_{0} \in \mathcal{Y}$ and input disturbance $u_{0} \in \mathcal{U}$, which are uncertainties. The standard feedback configuration is shown in Fig. 1, 


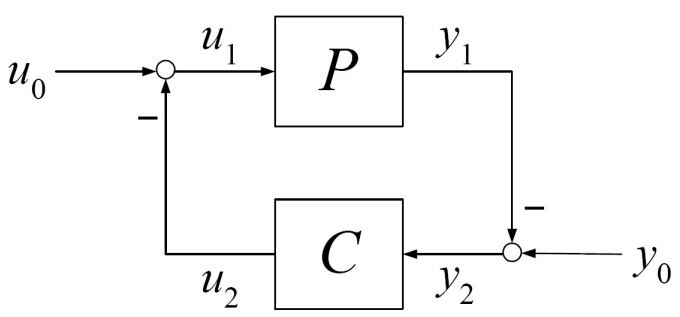

Fig. 1. Standard Feedback Configuration

and the signals have relations:

$$
\begin{array}{ll}
y_{1}=\mathcal{P} u_{1}, & u_{2}=\mathcal{C} y_{2} \\
y_{0}=y_{1}+y_{2}, & u_{0}=u_{1}+u_{2}
\end{array}
$$

Let $\mathcal{W}$ denote $\mathcal{U} \times \mathcal{Y}$, and write

$$
w_{0}=\left(\begin{array}{c}
u_{0} \\
y_{0}
\end{array}\right), \quad w_{1}=\left(\begin{array}{l}
u_{1} \\
y_{1}
\end{array}\right), \quad w_{2}=\left(\begin{array}{l}
u_{2} \\
y_{2}
\end{array}\right)
$$

Thus the closed-loop operator is defined by

$$
H_{\mathcal{P}, \mathcal{C}}: \mathcal{W} \rightarrow \mathcal{W} \times \mathcal{W}: w_{0} \mapsto\left(w_{1}, w_{2}\right)
$$

The graph of the plant $\mathcal{P}$ is defined as

$$
\mathcal{G}_{\mathcal{P}}=\left\{\left(\begin{array}{c}
u \\
\mathcal{P} u
\end{array}\right): u \in \mathcal{U}\right\} \subset \mathcal{W}
$$

and the graph of the control operator $\mathcal{C}$ is defined as

$$
\mathcal{G}_{\mathcal{C}}=\left\{\left(\begin{array}{c}
\mathcal{C} y \\
y
\end{array}\right): y \in \mathcal{Y}\right\} \subset \mathcal{W}
$$

For simplicity of notation, normally, we write $\mathcal{G}_{\mathcal{P}}$ as $\mathcal{M}$, and $\mathcal{G}_{\mathcal{C}}$ as $\mathcal{N}$ respectively.

To define robust stability of the closed-loop to disturbances, let $\Pi_{i}$ be the natural projection of $\mathcal{W} \times \mathcal{W}$ onto the $i$ th component ( $i=1,2)$, another tow operators

$$
\Pi_{\mathcal{M} / / \mathcal{N}}=\Pi_{1} H_{\mathcal{P}, \mathcal{C}}, \quad \Pi_{\mathcal{N} / / \mathcal{M}}=\Pi_{2} H_{\mathcal{P}, \mathcal{C}}
$$

are introduced, that is

$$
\begin{aligned}
& \Pi_{\mathcal{M} / \mathcal{N}}: \mathcal{W} \rightarrow \mathcal{W}: w_{0} \mapsto w_{1} \\
& \Pi_{\mathcal{N} / / \mathcal{M}}: \mathcal{W} \rightarrow \mathcal{W}: w_{0} \mapsto w_{2}
\end{aligned}
$$

Definition 1: The closed-loop $[\mathcal{P}, \mathcal{C}]$ is said to be stable if the operator $\Pi_{\mathcal{M} / / \mathcal{N}}$ has a finite induced norm, i.e.

$$
\left\|\Pi_{\mathcal{M} / / \mathcal{N}}\right\|=\sup _{w_{0} \neq 0} \frac{\left\|\Pi_{\mathcal{M} / / \mathcal{N}} w_{0}\right\|}{\left\|w_{0}\right\|}=\sup _{w_{0} \neq 0} \frac{\left\|w_{1}\right\|}{\left\|w_{0}\right\|}<\infty
$$

The notion of stability for nonlinear control is a strong requirement. It can be relaxed to gain-function stability as follows.

Definition 2: The gain-function of the operator $\Pi_{\mathcal{M} / / \mathcal{N}}$ is defined as

$$
g\left[\Pi_{\mathcal{M} / / \mathcal{N}}\right](\alpha)=\sup _{\left\|w_{0}\right\| \leq \alpha}\left\|\Pi_{\mathcal{M} / / \mathcal{N}} w_{0}\right\|, \alpha \geq 0
$$

The closed-loop $[\mathcal{P}, \mathcal{C}]$ is said to be gain-function ( gf- ) stable if $g\left[\Pi_{\mathcal{M} / \mathcal{N}}\right](\alpha)$ remains finite for all $\alpha \geq 0$.

It can be seen that if there exists a positive constant $\Gamma$ such that

$$
\left\|w_{1}\right\| \leq \Gamma\left\|w_{0}\right\|, w_{1}, w_{0} \in \mathcal{W}
$$

then $[\mathcal{P}, \mathcal{C}]$ is stable; if there exists a continuous function $\gamma(\cdot)>0$ such that

$$
\left\|w_{1}\right\| \leq \gamma\left(\left\|w_{0}\right\|\right), w_{1}, w_{0} \in \mathcal{W}
$$

then $[\mathcal{P}, \mathcal{C}]$ is gf-stable.

Finally, we define the gap metric between the nominal plant $\mathcal{P}$ and any perturbed plant $\mathcal{P}_{1}$ as follows.

Definition 3: For plant $\mathcal{P}$ and plant $\mathcal{P}_{1}$, the directed gap metric between the two plants is defined as

$$
\vec{\delta}\left(\mathcal{P}, \mathcal{P}_{1}\right)= \begin{cases}\inf _{\Phi \in \mathcal{O}}\left\|\left.(\Phi-I)\right|_{\mathcal{M}}\right\|, & \text { if } \mathcal{O} \neq \varnothing \\ \infty, & \text { if } \mathcal{O}=\varnothing\end{cases}
$$

and the gap metric is defined as

$$
\delta\left(\mathcal{P}, \mathcal{P}_{1}\right)=\max \left\{\vec{\delta}\left(\mathcal{P}, \mathcal{P}_{1}\right), \vec{\delta}\left(\mathcal{P}_{1}, \mathcal{P}\right)\right\}
$$

where

$$
\mathcal{O}=\left\{\Phi: \mathcal{M} \rightarrow \mathcal{M}_{1} \mid \Phi \text { is causal, bijective and } \Phi(0)=0\right\}
$$

and $\mathcal{M}_{1}$ is the graph of $\mathcal{P}_{1}$, namely $\mathcal{G}_{\mathcal{P}_{1}}$.

The significance of the introduction of gap metric lies in the following theorem.

Theorem 1: For the feedback system in Fig. 1, let the closed-loop $[\mathcal{P}, \mathcal{C}]$ be stable. If a plant $\mathcal{P}_{1}$ is such that

$$
\vec{\delta}\left(\mathcal{P}, \mathcal{P}_{1}\right)<\frac{1}{\left\|\Pi_{\mathcal{M} / / \mathcal{N}}\right\|}
$$

then the closed-loop $\left[\mathcal{P}_{1}, \mathcal{C}\right]$ is also stable, and

$$
\left\|\Pi_{\mathcal{M}_{1} / / \mathcal{N}}\right\| \leq\left\|\Pi_{\mathcal{M} / / \mathcal{N}}\right\| \frac{1+\vec{\delta}\left(\mathcal{P}, \mathcal{P}_{1}\right)}{1-\left\|\Pi_{\mathcal{M} / / \mathcal{N}}\right\| \vec{\delta}\left(\mathcal{P}, \mathcal{P}_{1}\right)}
$$

The proof of this theorem can be found in paper [4].

Definition 1, 2, and Theorem 1 provide a complete framework to design a robust controller in the presence of input, measurement disturbances, and plant perturbations. First, for the nominal plant $\mathcal{P}$ design a controller $\mathcal{C}$ such that the closed-loop operator $H_{\mathcal{P}, \mathcal{C}}$ is stable, namely, the operator $\Pi_{\mathcal{M}_{1} / / \mathcal{N}}$ has a finite induce norm, or the signals satisfy (1). Second, by Theorem 1, we get a plant robust margin $\left\|\Pi_{\mathcal{M}_{1} / / \mathcal{N}}\right\|^{-1}$, the controller $\mathcal{C}$ thus can stabilize any closedloop $\left[\mathcal{P}_{1}, \mathcal{C}\right]$ if the directed gap metric $\vec{\delta}\left(\mathcal{P}, \mathcal{P}_{1}\right)$ is properly small. The idea to design a robust gf-stable controller is similar. 


\section{B. Problem Formulation}

Consider following nominal plant in normal form

$$
\begin{aligned}
\mathcal{P}\left(x_{1}^{0}\right): \dot{x}_{11} & =x_{12} \\
& \vdots \\
\dot{x}_{1(n-1)} & =x_{1 n} \\
\dot{x}_{1 n} & =\varphi\left(x_{11}, \cdots, x_{1 n}\right)+u_{1} \\
y_{1} & =x_{11}
\end{aligned}
$$

where

$$
x_{1}^{0}=\left(x_{11}^{0}, \cdots, x_{1 n}^{0}\right)^{T}
$$

is the initial condition. Well-posedness of the differential equations grantees that of the feedback configuration, hence, for the well-posedness of the differential equations, we assume that $\varphi(0)=0$, and $\varphi$ is Lipschitz continuous, that is, there exist a constant $L$ such that for any $\omega_{1}, \omega_{2} \in \mathbb{R}^{n}$, it hold

$$
\left|\varphi\left(\omega_{1}\right)-\varphi\left(\omega_{2}\right)\right| \leq L\left\|\omega_{1}-\omega_{2}\right\|
$$

where we utilize $\|\cdot\|$ to denote the Euclid norm.

We introduce following notations

$$
\begin{gathered}
x_{1}=\left(\begin{array}{c}
x_{11} \\
x_{12} \\
\vdots \\
x_{1 n}
\end{array}\right), A=\left(\begin{array}{cccccc}
0 & 1 & 0 & \cdots & 0 & 0 \\
0 & 0 & 1 & \cdots & 0 & 0 \\
\cdots & \ldots & \ldots & \ldots & \ldots & \ldots \\
0 & 0 & 0 & \cdots & 0 & 1 \\
0 & 0 & 0 & \cdots & 0 & 0
\end{array}\right), B=\left(\begin{array}{c}
0 \\
\vdots \\
0 \\
1
\end{array}\right) \\
C=(1,0, \cdots, 0)
\end{gathered}
$$

then the plant can be rewrite as

$$
\begin{aligned}
\mathcal{P}\left(x_{1}^{0}\right): \dot{x}_{1} & =A x_{1}+B\left(\varphi\left(x_{1}\right)+u_{1}\right), x_{1}(0)=x_{1}^{0} \\
y_{1} & =C x_{1}
\end{aligned}
$$

Take signal spaces

$$
\mathcal{U}=L^{\infty}(\mathbb{R}), \quad \mathcal{Y}=L^{\infty}(\mathbb{R})
$$

then

$$
\mathcal{P}\left(x_{1}^{0}\right): L^{\infty}(\mathbb{R}) \rightarrow L^{\infty}(\mathbb{R}): u_{1} \mapsto y_{1}
$$

The norm for the space $L^{\infty}(\mathbb{R})$ is defined as the normal norm $\|\cdot\|_{\infty}$.

The problem is to design a controller such that the closedloop system is stable or gf-stable. Alternatively, such that there exist a constant $\Gamma$ and a gain function $\gamma(\cdot)$ which satisfy (1) and (2).

\section{DESIGN OF CONTROLLER}

Firstly, we chose a vector

$$
K=\left(k_{1}, \cdots, k_{n}\right)
$$

such that $A_{0}=A+B K$ is Hurwitz. Secondly, we chose positive constants $\alpha_{i}, i=1, \cdots, n$ such that the roots of the equation

$$
s^{n}+\alpha_{1} s^{n-1}+\cdots+\alpha_{n-1} s+\alpha_{n}=0
$$

are in the left-half plane, and let

$$
D=\left(\alpha_{1}, \alpha_{2}, \cdots, \alpha_{n}\right)^{T}
$$

Then we design an observer as

$$
\begin{aligned}
\dot{\hat{x}}_{2} & =A \hat{x}_{2}-D\left(y_{2}-\hat{y}_{2}\right)+B K \hat{x}_{2}, \hat{x}_{2}(0)=\hat{x}_{2}^{0} \\
\hat{y}_{2} & =C \hat{x}_{2}
\end{aligned}
$$

Lastly, we define a controller as

$$
\begin{aligned}
\mathcal{C}\left(\hat{x}_{2}^{0}\right): & \\
u_{2} & =\varphi\left(-y_{2}\right)+K \hat{x}_{2} \\
\dot{\hat{x}}_{2} & =A \hat{x}_{2}+D\left(y_{2}-\hat{y}_{2}\right)+B K \hat{x}_{2}, \hat{x}_{2}(0)=\hat{x}_{2}^{0} \\
\hat{y}_{2} & =C \hat{x}_{2}
\end{aligned}
$$

\section{Robustness AnAlysis}

First we prove a lemma about the estimate of the observer error.

Lemma 1: Let $x_{1}$ be the state of the plant in (4), and $\hat{x}_{2}$ be observer state in (5), and let

$$
\tilde{x}=x_{1}+\hat{x}_{2}
$$

be the perturbed observer error. Then there exist positive constants $b$ and $\beta$ such that

where

$$
\|\tilde{x}\|_{\infty} \leq b\left\|\tilde{x}^{0}\right\|+\beta\left\|\left(u_{0}, y_{0}\right)^{T}\right\|_{\infty}
$$

$$
\tilde{x}^{0}=x_{1}^{0}+\hat{x}_{2}^{0}
$$

Proof: Note that

$$
u_{1}=u_{0}-u_{2}, \quad y_{2}=y_{0}-y_{1}
$$

then the closed-loop $\left[\mathcal{P}\left(x_{1}^{0}\right), \mathcal{C}\left(\hat{x}_{2}^{0}\right)\right]$ can be written as

$\dot{x}_{1}=A x_{1}+B\left(\varphi\left(y_{1}\right)-\varphi\left(-y_{2}\right)-K \hat{x}_{2}+u_{0}\right), x_{1}(0)=x_{1}^{0}$

$\dot{\hat{x}}_{2}=A \hat{x}_{2}+D\left(y_{0}-C\left(x_{1}+\hat{x}_{2}\right)\right)+B K \hat{x}_{2}, \quad \hat{x}_{2}(0)=\hat{x}_{2}^{0}$

From above two equations, we obtain

$$
\dot{\tilde{x}}=A_{1} \tilde{x}+D y_{0}+B\left(\varphi\left(y_{1}\right)-\varphi\left(-y_{2}\right)+u_{0}\right), \tilde{x}(0)=\tilde{x}^{0}
$$

where

$$
A_{1}=A-D C
$$

and it can be verified that the matrix $A_{1}$ is Hurwitz. 
Solving (8), we obtain

$$
\begin{aligned}
\tilde{x}= & e^{A_{1} t} \tilde{x}^{0}+\int_{0}^{t} e^{A_{1}(t-\tau)} \times \\
& \left(D y_{0}(\tau)+B\left(\varphi\left(y_{1}(\tau)\right)-\varphi\left(-y_{2}(\tau)\right)+u_{0}(\tau)\right)\right) d \tau
\end{aligned}
$$

Since $A_{1}$ is Hurwitz, all the real parts of the eigenvalues of $A_{1}$ are negative. We take a positive constant $\mu$ such that $-\mu$ is greater than all the real parts of the eigenvalues of $A_{1}$, then there exists a positive constant $b$ such that

$$
\left\|e^{A_{1} t}\right\| \leq b e^{-\mu t}
$$

On the other hand, by Lipschitz condition, it holds that

$$
\left|\varphi\left(y_{1}\right)-\varphi\left(-y_{2}\right)\right| \leq L\left|y_{1}+y_{2}\right|=L\left|y_{0}\right|
$$

Therefore, from (9), we obtain

$$
\begin{aligned}
& \|\tilde{x}(t)\| \\
\leq & \left\|\tilde{x}^{0}\right\| \cdot\left\|e^{A_{1} t}\right\|+\int_{0}^{t} d \tau\left\|e^{A_{1}(t-\tau)}\right\| \times \\
& \left(\left\|D y_{0}(\tau)\right\|+\left(\left\|\varphi\left(y_{1}(\tau)\right)-\varphi\left(-y_{2}(\tau)\right)\right\|\right)+\left\|u_{0}(\tau)\right\|\right) \\
\leq & \left\|\tilde{x}^{0}\right\| b e^{-\mu t} \\
& +\int_{0}^{t} b e^{-\mu(t-\tau)}\left((\|D\|+L)\left\|y_{0}\right\|_{\infty}+\left\|u_{0}\right\|_{\infty}\right) d \tau \\
\leq & b\left\|\tilde{x}^{0}\right\|+\frac{b}{\mu}\left((\|D\|+L)\left\|y_{0}\right\|_{\infty}+\left\|u_{0}\right\|_{\infty}\right) \\
\leq & b\left\|\tilde{x}^{0}\right\|+\beta\left\|\left(u_{0}, y_{0}\right)^{T}\right\|_{\infty}
\end{aligned}
$$

Write

$$
\beta=\frac{b \sqrt{2}}{\mu} \max \{\|D\|+L, 1\}
$$

then we obtain (7), and complete the proof.

Now we state and prove the main result.

Theorem 2: Let the plant $\mathcal{P}\left(x_{1}^{0}\right)$ and controller $\mathcal{C}\left(\hat{x}_{2}^{0}\right)$ be defined by (3) and (6). Then

1) There exists a continuous function $\gamma: \mathbb{R}_{+}^{3} \rightarrow[0,+\infty)$ such that for all $\left(u_{0}, y_{0}\right)^{T} \in L^{\infty}\left(\mathbb{R}^{+}\right) \times L^{\infty}\left(\mathbb{R}^{+}\right)$, it holds

$$
\left\|\left(u_{1}, y_{1}\right)^{T}\right\|_{\infty} \leq \gamma\left(\left\|\left(u_{0}, y_{0}\right)^{T}\right\|_{\infty},\left\|\tilde{x}_{0}\right\|,\left\|x_{1}^{0}\right\|_{\infty}\right)
$$

that is, the closed-loop $\left[\mathcal{P}\left(x_{1}^{0}\right), \mathcal{C}\left(\hat{x}_{2}^{0}\right)\right]$ is gf-stable.

2) If $x_{1}^{0}$ is zero, by setting $\hat{x}_{2}^{0}$ to be zero, there exists a positive constant $\Gamma$ such that for all $\left(u_{0}, y_{0}\right)^{T} \in L^{\infty}\left(\mathbb{R}^{+}\right) \times L^{\infty}\left(\mathbb{R}^{+}\right)$, it holds

$$
\left\|\left(u_{1}, y_{1}\right)^{T}\right\|_{\infty} \leq \Gamma\left\|\left(u_{0}, y_{0}\right)^{T}\right\|_{\infty}
$$

that is, the closed-loop $[\mathcal{P}(0), \mathcal{C}(0)]$ is stable.

Proof: Let $Q$ be the solution of the equation

$$
(A+B K)^{T} Q+Q(A+B K)=-2 I
$$

and consider the Lyapunov function

$$
V(t)=V\left(x_{1}\right)(t)=x_{1}^{T} Q x_{1}
$$

then along the trajectories of the closed-loop, we have

$$
\begin{aligned}
\dot{V}= & \dot{x}_{1}^{T} Q x_{1}+x_{1}^{T} Q \dot{x}_{1} \\
= & \left(A x_{1}+B\left(\varphi\left(y_{1}\right)+u_{1}\right)\right)^{T} Q x_{1} \\
& +x_{1}^{T} Q\left(A x_{1}+B\left(\varphi\left(y_{1}\right)+u_{1}\right)\right) \\
= & \left(A x_{1}+B\left(\varphi\left(y_{1}\right)+u_{0}-u_{2}\right)\right)^{T} Q x_{1} \\
& +x_{1}^{T} Q\left(A x_{1}+B\left(\varphi\left(y_{1}\right)+u_{0}-u_{2}\right)\right) \\
= & \left(A x_{1}+B\left(\varphi\left(y_{1}\right)+u_{0}-\varphi\left(-y_{2}\right)-K \hat{x}_{2}\right)\right)^{T} Q x_{1} \\
& +x_{1}^{T} Q\left(A x_{1}+B\left(\varphi\left(y_{1}\right)+u_{0}-\varphi\left(-y_{2}\right)-K \hat{x}_{2}\right)\right) \\
= & x_{1}^{T}\left((A+B K)^{T} Q+Q(A+B K)\right) x_{1} \\
& +2 B^{T} Q x_{1}\left(\varphi\left(y_{1}\right)-\varphi\left(-y_{2}\right)-K \tilde{x}+u_{0}\right) \\
= & -2 x_{1}^{T} x_{1}+2 B^{T} Q x_{1}\left(\varphi\left(y_{1}\right)-\varphi\left(-y_{2}\right)-K \tilde{x}+u_{0}\right) \\
= & -2\left\|x_{1}\right\|^{2}+2 B^{T} Q x_{1}\left(\varphi\left(y_{1}\right)-\varphi\left(-y_{2}\right)-K \tilde{x}+u_{0}\right)
\end{aligned}
$$

Let

$$
Q=\left\{q_{i j}\right\}_{n \times n}
$$

and

$$
q_{1}=\max _{1 \leq j \leq n}\left\{\left|q_{1 j}\right|\right\}
$$

then

$$
B^{T} Q x_{1} \leq q_{1}\left\|x_{1}\right\|
$$

On the other hand, from the Lipschitz condition and Lemma 1 , we obtain

$$
\begin{aligned}
& \varphi\left(y_{1}\right)-\varphi\left(-y_{2}\right)-K \tilde{x}+u_{0} \\
\leq & L\left\|y_{0}\right\|_{\infty}+\|K\|\|\tilde{x}\|_{\infty}+\left\|u_{0}\right\|_{\infty} \\
\leq & l \sqrt{2}\left\|\left(u_{0}, y_{0}\right)^{T}\right\|_{\infty}+\|K\|\left(b\left\|\tilde{x}^{0}\right\|+\beta\left\|\left(u_{0}, y_{0}\right)^{T}\right\|_{\infty}\right) \\
\leq & b^{*}\left\|\tilde{x}^{0}\right\|+\beta^{*}\left\|\left(u_{0}, y_{0}\right)^{T}\right\|_{\infty}
\end{aligned}
$$

where

$$
\begin{gathered}
l=\max \{L, 1\} \\
b^{*}=\|K\| b \\
\beta^{*}=l \sqrt{2}+\|K\| \beta
\end{gathered}
$$

Hence

$$
\begin{aligned}
& 2 B^{T} Q x_{1}\left(\varphi\left(y_{1}\right)-\varphi\left(-y_{2}\right)-K \tilde{x}+u_{0}\right) \\
\leq & 2 q_{1}\left(b^{*}\left\|\tilde{x}^{0}\right\|+\beta^{*}\left\|\left(u_{0}, y_{0}\right)^{T}\right\|_{\infty}\right)\left\|x_{1}\right\|
\end{aligned}
$$

Therefore

$$
\begin{aligned}
\dot{V}= & -2\left\|x_{1}\right\|^{2}+2 B^{T} Q x_{1}\left(\varphi\left(y_{1}\right)-\varphi\left(-y_{2}\right)-k \tilde{x}+u_{0}\right) \\
\leq & -\left\|x_{1}\right\|^{2}-\left\|x_{1}\right\|^{2} \\
& +2 q_{1}\left(b^{*}\left\|\tilde{x}^{0}\right\|+\beta^{*}\left\|\left(u_{0}, y_{0}\right)^{T}\right\|_{\infty}\right)\left\|x_{1}\right\|
\end{aligned}
$$


By Young's Inequality, we obtain that

$$
\dot{V} \leq-\left\|x_{1}\right\|^{2}+q_{1}^{2}\left(b^{*}\left\|\tilde{x}^{0}\right\|+\beta^{*}\left\|\left(u_{0}, y_{0}\right)^{T}\right\|_{\infty}\right)^{2}
$$

then $V$ decreases monotonically outside the compact set

$$
\mathbf{R}=\left\{x_{1} \in \mathbb{R}^{n} \mid\left\|x_{1}\right\| \leq q_{1}\left(b^{*}\left\|\tilde{x}^{0}\right\|+\beta^{*}\left\|\left(u_{0}, y_{0}\right)^{T}\right\|_{\infty}\right)\right\}
$$

So, if

$$
x_{1}^{0} \leq b^{*}\left\|\tilde{x}^{0}\right\|+\beta^{*}\left\|\left(u_{0}, y_{0}\right)^{T}\right\|_{\infty}
$$

then $V(t)$ remains in $\mathbf{R}$; if

$$
x_{1}^{0}>b^{*}\left\|\tilde{x}^{0}\right\|+\beta^{*}\left\|\left(u_{0}, y_{0}\right)^{T}\right\|_{\infty}
$$

then $V(t)$ monotonically decreases from $t=0$ until $x_{1}$ reaches $\mathbf{R}$. Therefore

$$
V(t) \leq \max \left\{V\left(x_{1}^{0}\right), \bar{V}\right\}
$$

where

$$
\bar{V}=\sup \left\{V\left(x_{1}\right) \mid\left\|x_{1}\right\| \leq q_{1}\left(b^{*}\left\|\tilde{x}^{0}\right\|+\beta^{*}\left\|\left(u_{0}, y_{0}\right)^{T}\right\|_{\infty}\right)\right\}
$$

Note that

$$
\underline{\lambda}(Q)\left\|x_{1}\right\|^{2} \leq V\left(x_{1}\right) \leq \bar{\lambda}(Q)\left\|x_{1}\right\|^{2}
$$

where $\bar{\lambda}(Q), \underline{\lambda}(Q)$ are the maximum and minim eigenvalues of matrix $Q$ respectively, then

$$
\begin{aligned}
& \sup \left\{V\left(x_{1}\right) \mid\left\|x_{1}\right\| \leq q_{1}\left(b^{*}\left\|\tilde{x}^{0}\right\|+\beta^{*}\left\|\left(u_{0}, y_{0}\right)^{T}\right\|_{\infty}\right)\right\} \\
= & \sup \times \\
& \left\{\bar{\lambda}(Q)\left\|x_{1}\right\|^{2} \mid\left\|x_{1}\right\| \leq q_{1}\left(b^{*}\left\|\tilde{x}^{0}\right\|+\beta^{*}\left\|\left(u_{0}, y_{0}\right)^{T}\right\|_{\infty}\right)\right\} \\
= & \bar{\lambda}(Q)\left(b^{*}\left\|\tilde{x}^{0}\right\|+\beta^{*}\left\|\left(u_{0}, y_{0}\right)^{T}\right\|_{\infty}\right)^{2}
\end{aligned}
$$

therefore

$$
\left\|x_{1}\right\| \leq \sqrt{\frac{V}{\underline{\lambda}(Q)}} \leq \max \left\{\sqrt{\frac{\bar{\lambda}(Q)}{\underline{\lambda}(Q)}}\left\|x_{1}^{0}\right\|, X\right\}
$$

where

$$
X=q_{1} \sqrt{\bar{\lambda}(Q)}\left(b^{*}\left\|\tilde{x}^{0}\right\|+\beta^{*}\left\|\left(u_{0}, y_{0}\right)^{T}\right\|_{\infty}\right)
$$

Write

$$
g(p, q, r)=\max \left\{\sqrt{\frac{\bar{\lambda}(Q)}{\underline{\lambda}(Q)}} r, q_{1} \sqrt{\bar{\lambda}(Q)}\left(b^{*} q+\beta^{*} p\right)\right\}
$$

then the above inequality can be rewritten as

$$
\left\|x_{1}\right\| \leq g\left(\left\|\left(u_{0}, y_{0}\right)^{T}\right\|_{\infty},\left\|\tilde{x}^{0}\right\|,\left\|x_{1}^{0}\right\|\right)
$$

So

$$
\left\|x_{1}\right\|_{\infty} \leq g\left(\left\|\left(u_{0}, y_{0}\right)^{T}\right\|_{\infty},\left\|\tilde{x}^{0}\right\|,\left\|x_{1}^{0}\right\|\right)
$$

since the left hand side of the inequality is a constant.

Therefore we obtain our estimate for $\left\|y_{1}\right\|_{\infty}$ as follows.

$$
\begin{aligned}
\left\|y_{1}\right\|_{\infty} & =\left\|x_{11}\right\|_{\infty} \leq\left\|x_{1}\right\|_{\infty} \\
& \leq g\left(\left\|\left(u_{0}, y_{0}\right)^{T}\right\|_{\infty},\left\|\tilde{x}^{0}\right\|,\left\|x_{1}^{0}\right\|\right)
\end{aligned}
$$

Next we estimate $\left\|u_{1}\right\|_{\infty}$. First

$$
\begin{aligned}
u_{1} & =u_{0}-u_{2} \\
& =u_{0}-\varphi\left(-y_{2}\right)-K \hat{x}_{2} \\
& =u_{0}+\varphi\left(y_{1}\right)-\varphi\left(-y_{2}\right)-K\left(x_{1}+\hat{x}_{2}\right)-\varphi\left(y_{1}\right)+K x_{1} \\
& =u_{0}+\varphi\left(y_{1}\right)-\varphi\left(-y_{2}\right)-K \tilde{x}-\varphi\left(y_{1}\right)+K x_{1}
\end{aligned}
$$

Note that $\varphi$ is Lipschitz, and $\varphi(0)$ is zero, hence

$$
\begin{aligned}
\left\|u_{1}\right\|_{\infty} \leq & \left\|u_{0}\right\|_{\infty}+\left\|\varphi\left(y_{1}\right)-\varphi\left(-y_{2}\right)\right\|_{\infty}+\|K\|\|\tilde{x}\|_{\infty} \\
& +\left\|\varphi\left(y_{1}\right)\right\|_{\infty}+\|K\|\left\|x_{1}\right\|_{\infty} \\
\leq & \left\|u_{0}\right\|_{\infty}+L\left\|y_{1}+y_{2}\right\|_{\infty}+\|K\|\|\tilde{x}\|_{\infty} \\
& +L\left\|y_{1}\right\|_{\infty}+\|K\|\left\|x_{1}\right\|_{\infty} \\
\leq & \left\|u_{0}\right\|_{\infty}+L\left\|y_{0}\right\|_{\infty} \\
& +\|K\|\left(b\left\|\tilde{x}^{0}\right\|+\beta\left\|\left(u_{0}, y_{0}\right)^{T}\right\|_{\infty}\right) \\
& +L g\left(\left\|\left(u_{0}, y_{0}\right)^{T}\right\|_{\infty},\left\|\tilde{x}^{0}\right\|,\left\|x_{1}^{0}\right\|\right) \\
& +\|K\| g\left(\left\|\left(u_{0}, y_{0}\right)^{T}\right\|_{\infty},\left\|\tilde{x}^{0}\right\|,\left\|x_{1}^{0}\right\|\right) \\
\leq & \sqrt{2}\left\|\left(u_{0}, y_{0}\right)^{T}\right\|_{\infty} \\
& +\|K\|\left(b\left\|\tilde{x}^{0}\right\|+\beta\left\|\left(u_{0}, y_{0}\right)^{T}\right\|_{\infty}\right) \\
& +(L+\|K\|) g\left(\left\|\left(u_{0}, y_{0}\right)^{T}\right\|_{\infty},\left\|\tilde{x}^{0}\right\|,\left\|x_{1}^{0}\right\|\right)
\end{aligned}
$$

Write

$$
h(p, q, r)=l \sqrt{2} p+\|K\|(b q+\beta p)+(L+\|K\|) g(p, q, r)
$$

then we obtain

$$
\left\|u_{1}\right\|_{\infty} \leq h\left(\left\|\left(u_{0}, y_{0}\right)^{T}\right\|_{\infty},\left\|\tilde{x}^{0}\right\|,\left\|x_{1}^{0}\right\|\right)
$$

Therefore, write

$$
\gamma(p, q, r)=\left(g(p, q, r)^{2}+h(p, q, r)^{2}\right)^{\frac{1}{2}}
$$

then we have built up the following inequality

$$
\begin{aligned}
\left\|\left(u_{1}, y_{1}\right)^{T}\right\|_{\infty}= & \left(\left\|u_{1}\right\|_{\infty}^{2}+\left\|u_{1}\right\|_{\infty}^{2}\right)^{\frac{1}{2}} \\
\leq & {\left[g\left(\left\|\left(u_{0}, y_{0}\right)^{T}\right\|_{\infty},\left\|\tilde{x}^{0}\right\|,\left\|x_{1}^{0}\right\|\right)^{2}\right.} \\
& \left.+h\left(\left\|\left(u_{0}, y_{0}\right)^{T}\right\|_{\infty},\left\|\tilde{x}^{0}\right\|,\left\|x_{1}^{0}\right\|\right)^{2}\right]^{\frac{1}{2}} \\
= & \gamma\left(\left\|\left(u_{0}, y_{0}\right)^{T}\right\|_{\infty},\left\|\tilde{x}^{0}\right\|,\left\|x_{1}^{0}\right\|\right)
\end{aligned}
$$

that is, the closed-loop is gf-stable.

If $x_{1}^{0}=0$ and $\hat{x}_{2}^{0}=0$, then $\tilde{x}^{0}=0$. From the definitions of functions $g$ and $h$

$$
g(p, 0,0)=q_{1} \beta^{*} \sqrt{\bar{\lambda}(Q)} p
$$

hence

$$
\begin{aligned}
h(p, 0,0) & =(l \sqrt{2}+\|k\| \beta) p+(L+\|k\|) g(p, 0,0) \\
& =(l \sqrt{2}+\|k\| \beta) p+(L+\|k\|) q_{1} \beta^{*} p \\
& =\left(l \sqrt{2}+\|k\| \beta+(L+\|k\|) q_{1} \beta^{*} \sqrt{\bar{\lambda}(Q)}\right) p
\end{aligned}
$$




$$
\begin{aligned}
& \gamma(p, 0,0) \\
= & \left(g(p, 0,0)^{2}+h(p, 0,0)^{2}\right)^{\frac{1}{2}} \\
= & {\left[\left(q_{1} \beta^{*} \sqrt{\bar{\lambda}(Q) p}\right)^{2}\right.} \\
& \left.+\left(\left(l \sqrt{2}+\|k\| \beta+(L+\|k\|) q_{1} \beta^{*} \sqrt{\bar{\lambda}(Q)}\right) p\right)^{2}\right]^{\frac{1}{2}} \\
= & {\left[\left(q_{1}^{2}\left(\beta^{*}\right)^{2} \bar{\lambda}(Q)\right.\right.} \\
& \left.+\left(l \sqrt{2}+\|k\| \beta+(L+\|k\|) q_{1} \beta^{*} \sqrt{\bar{\lambda}(Q)}\right)^{2}\right]^{\frac{1}{2}} p
\end{aligned}
$$

Let

$$
\begin{aligned}
\Gamma= & {\left[\left(q_{1}^{2}\left(\beta^{*}\right)^{2} \bar{\lambda}(Q)\right.\right.} \\
& \left.+\left(l \sqrt{2}+\|k\| \beta+(L+\|k\|) q_{1} \beta^{*} \sqrt{\bar{\lambda}(Q)}\right)^{2}\right]^{\frac{1}{2}}
\end{aligned}
$$

then, it follows that (11) holds.

A robustness to plant perturbations can be given as follows.

Theorem 3: Let the plant $\mathcal{P}\left(x_{1}^{0}\right)$ and controller $\mathcal{C}\left(\hat{x}_{2}^{0}\right)$ be defined by (3) and (6). Then there exists $\Gamma>0$ such that if a plant $\mathcal{P}_{1}$ satisfies

$$
\vec{\delta}\left(\mathcal{P}(0), \mathcal{P}_{1}\right)<\frac{1}{\Gamma}
$$

then the closed-loop $\left[\mathcal{P}_{1}, \mathcal{C}(0)\right]$ is also stable, and

$$
\left\|\Pi_{\mathcal{M}_{1} / / \mathcal{N}}\right\| \leq \Gamma \frac{1+\vec{\delta}\left(\mathcal{P}(0), \mathcal{P}_{1}\right)}{1-\Gamma \vec{\delta}\left(\mathcal{P}(0), \mathcal{P}_{1}\right)}
$$

Proof: By Theorem 2, we have shown that there exists $\Gamma>0$ such that

$$
\left\|\Pi_{\mathcal{M} / / \mathcal{N}}\right\| \leq \Gamma
$$

Then, if

$$
\vec{\delta}\left(\mathcal{P}, \mathcal{P}_{1}\right)<\frac{1}{\Gamma}
$$

it holds that

$$
\vec{\delta}\left(\mathcal{P}, \mathcal{P}_{1}\right)<\frac{1}{\left\|\Pi_{\mathcal{M} / / \mathcal{N}}\right\|}
$$

Hence, by Theorem 1 , the closed-loop $\left[\mathcal{P}_{1}, \mathcal{C}(0)\right]$ is stable, and (14) holds, and the proof is completed.
Within the framework of nonlinear gap metric, an output feedback control design procedure for robust stability has been established in the presence of input, measurement disturbances, and plant perturbations. Any restriction on input and measurement disturbances is not required. Unmodeled plant perturbations such as time delay are allowable uncertainties. So, the work in this paper shows that the framework of nonlinear gap metric is an appropriate topological structure for robust output feedback control designs. This paper therefore represents a start to apply the framework of nonlinear gap metric to output feedback control designs for robust stability.

A global Lipschitz condition is imposed on the nonlinearity of the nominal plant, which is for a global result of input and measurement disturbances; a relaxation of the requirement to local Lipschitz condition leads a semi-global results.

\section{REFERENCES}

[1] A. El-Sakkary, The gap metric: Robustness of stabilization of feedback systems, IEEE Transaction on Automatic Control 30 (1985), no. 3, 240-247.

[2] J. Gauthier, H. Hamouri, and I. Kupka, Observer for nonlinear systems, Proceedings of the 30th IEEE Conference on Decision and Control (Brighton, England), IEEE, December 1991, pp. 1483-1489.

[3] J. Gauthier and I. Kupka, Observerty and observer for nonlinear systems, SIAM Journal on Control and Optimization 32 (1994), no. 4, 975-994.

[4] T. Georgiou and M. Smith, Robustness analysis of nonlinear feedback systems: An input-output approach, IEEE Transactions on Automatic Control 42 (1997), no. 9, 1200-1221.

[5] A. Krener and A. Isidori, Linearization by output and non-linear observers, Systems \& Control Letters 3 (1983), no. 1, 47-52.

[6] R. Marino and P. Tomei, Global adaptive outputfeedback control of nonlinear systems, part 1: Linear parameterization, IEEE Transactions on Automatic Control 38 (1993), 17-32.

[7] C. Xie and M. French, Gap metric robustness of a backstepping control design, Proceedings of the 42nd IEEE Conference on Decision and Control (Hawaii, USA), vol. 5, December 2003, pp. 5180-5184.

[8] G. Zames and A. EI-Sakkary, Unstable systems and feedback: The gap metric, Proceedings of Allerton Conference (Allerton), October 1980, pp. 380-385. 